\title{
A Mini-Review on Candidate Genes for Regulation of Reproduction and Puberty Onset
}

\author{
Iffat Fatima*, Abdul-Aziz Khan and Zainab Rehman \\ Iffat Fatima, Department of Animal Sciences, Pakistan \\ *Corresponding author: Iffat Fatima, Laboratory of Physiology, Department of Animal Sciences, Pakistan
}

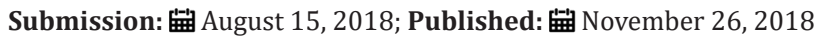

\begin{abstract}
Initiation and progression of puberty is a critical event for mammals. Various neuronal and hormonal cues are required for normal onset of puberty. GnRH is the major hormone involved in regulation of these mechanisms. Regulation of this complex process is controlled by multiple genes which encode respective proteins used as stimulatory inputs like glutamate, kisspeptin etc. or inhibitory inputs. These genes include KISS1/Kiss1, KISSR, Kissr, Tac3, TacR3, LEP, EAP1 etc. Findings of different studies are reviewed. Significance of Tac3 and TacR3 is evaluated by administering its agonist senktide in prepubertal female rats which results in increased LH secretion. Moreover, expression of Kiss1 gene is more sensitive to negative feedback effect of sex-steroids early in puberty than Tac2 expression. EAP1 is essential for normal menstrual cycle in female monkeys. LIN28A and LIN28B are considered essential for pubertal development and are good candidate genes for monogenic ICCP. Similarly, mutations in Leptin gene (LEP) result in abnormalities including obesity, delayed puberty and CDGP etc..
\end{abstract}

Keywords: Puberty genes; Hypogonadotropic hypogonadism (IHH); Kisspeptin

Abbreviations: IHH: Ideopathic Hypogonadotropic Hypogonadism; CCP: Central Precocious Puberty; ICCP: Ideopathic Central Precocious Puberty; CDGP: Constitutional Delay in Growth and Puberty; LEP: Leptin Gene

\section{Introduction}

The pulsatile release of GnRH from hypothalamus is essential for induction of initiation and progression of puberty in mammals. A network of neurons located in the medial basal hypothalamus $(\mathrm{MBH})$ in primates is the source of GnRH release into portal vessels which makes a connection between brain and pituitary gland. Alterations in neuronal and glial inputs to GnRH producing neurons control its periodic secretion. Regulatory mechanisms of this process at transcriptional level are not well understood, but it is apparent that several genes are involved [1]. Glutamate and kisspeptin signaling provides excitatory input whereas, (GABA) ergic and opiatergic neurons constitute inhibitory neuronal network. Glial cells mediate GnRH secretion via intercellular communication though various growth factors. Regulation of pubertal process by functionally linked genes provides the polygenetic evidence rather than specified by a single gene [2]. Evidences from various puberty related defects support the significance of the respective genes in puberty onset. These include GnRH1, GnRHR, KISS1, KISS1R, Tac3, TacR3, Eap1, Lin28, LH receptor gene and FSH receptor gene etc. It can be exemplified by loss of function mutations in KISSR in humans result in IHH whereas, gain of function mutations can lead to CPP (central precocious puberty) [3].

\section{Discussion}

Previous studies reveal that pubertal onset involves additive effect of various genes however monogenic basis of Ideopathic central precocious puberty is also suggested [4].

\section{Significance of KISS1/Kiss1 and KISSR/Kissr genes for puberty}

Kisspeptin has key role in the onset of puberty. It stimulates Gonadotropin releasing hormone (GnRH) from the GnRH neurons present in the hypothalamus. Kisspeptin works through its receptor named GpR54 [5]. Mutations in genes encoding Kisspeptin and its receptor provide the evidence for its role in puberty onset. This is revealed by infertility and hypogonadotropic hypogonadism resulted due to mutations in these genes. Moreover, exogenous administration of $\mathrm{GnRH}$ compensates the effect of mutation of KISS1R/kiss1r. Mayer et al. [6], found that in spite of ablation of Kiss1 expressing cells in brain of female mice, normal puberty onset and fertility is exhibited, challenging the significance of Kiss1 neurons for reproduction.

Differential requirement of Kiss1 expression for successful reproduction in male and female mice: Popa et al. [7], devised an experiment to prove the significance of kisspeptin genes, according to which normal reproduction can be supported by minute amount of kisspeptin. In this experiment mice (Kiss1-CreGFP) severely deficient in Kiss1 expression, were used. It was observed that female Kiss1/Cre/Cre mice showed reduced ovulation and impaired fertility whereas; male Kiss1Cre/Cre mice sired normalsized litters. Thus, it is concluded that females require higher levels of Kiss1 expression for competent reproduction as compared to males which require only $5 \%$ of normal Kiss 1 expression for normal reproduction. 
Evidence from mutation for importance of Kiss1 gene: Silveira et al. [3] reported the identification of a KISS1 mutation associated with male CPP (Central precocious puberty). It was found that kisspeptin resistance to in vitro degradation is increased which suggests that kisspeptin bioavailability is enhanced which may cause precocious puberty phenotype. Study was done on the role of KISS1 gene mutation in a boy attaining precocious puberty early at 1 year of age. Kisspeptin amino-acid sequencing revealed that proline in position 74 is very important in maintaining the kisspeptin structure. Mutation of proline at 74 position was noted in the subject. So, it suggests that p.P74S makes kisspeptin stable and less degradable due to which its bioavailability increases and CPP results. Mutations of KISS1 gene may cause GnRH-dependent disorders such as central precocious puberty and isolated hypogonadotropic hypogonadism (IHH). In females attaining CPP before 6yr of age, another mutation p.H90D was identified in the homozygous State. Mutations in KISS1 and KISS1R are not common causes of ICPP in girls.

\section{Tac3/TacR3}

Neurokinin B (NKB) and its receptor, neurokinin-3 receptor (NK3R), (encoded by Tac3 and TacR3 respectively) are considered essential elements for normal reproduction. Navarro et al. [8], performed an experiment on female rats for investigating the regulation of Tac 2 and Tacr3 mRNAs. The study revealed increased expression of both genes in brain (including lateral hypothalamic area and the arcuate nucleus) at puberty. It was also revealed that central induction of senktide (NK3R agonist) caused the LH secretion in prepubertal and peripubertal females. In contrast, delayed timing of vaginal opening and decreased LH secretion were found in association with chronic infusion of NK3R antagonist during puberty. These findings reveal the significance of Tac3 and Tac3R genes in regulation of puberty. Moreover, it was found that expression of these genes is affected by metabolic state at puberty i.e. after $48 \mathrm{hr}$ fasting Tacr3 expression is reduced. It was further revealed that vaginal opening is rescued (in 50\% animals) and LH secretion is increased in female rats with pubertal arrest (due to constant undernutrition), by repeated infusion of senktide (NKB agonist). Thus, it is suggested that NKB-NK3R signaling is significant for pubertal maturation and different pubertal disorders associated with under-nutrition and metabolic stress may arise due to changes in expression of genes encoding these proteins.

Expression of Tac2 as an early marker of puberty in mouse with differential sensitivity to steroidal negative feed-back effect than Kiss1: Gill et al. [9], measured the levels of Kiss1 and Tac2 mRNA in sex steroid deficient hpg and wild type mice. They found elevated level of Kiss1 mRNA in the arcuate nucleus of sex steroid deficient hpg mice while it remained constant in wild type mice. It reveals the negative feedback effect of sex steroids on Kiss1 expression early in development and throughout puberty. Conversely, both wild type and hpg mice showed elevated levels of Tac2 mRNA, encoding NKB, and its receptor (NK3R; encoded by Tacr3) across pubertal development. Thus, it is suggested that negative feedback effect of sex-steroid is lower for Tac2 as compared to Kiss1. Moreover, relative responsiveness of Kiss1and Tac2 was examined by evaluating the effect of estradiol (E2) on Kiss 1 and Tac2 expression. It was observed that at both juvenile and adult ages, inhibitory effect of estradiol was more pronounced for Kiss1 as compared to Tac2 expression. Finally, NK3R antagonist was administered to pre-pubertal females however, no effect was observed on pubertal markers in wild type and hpg mice. Thus, it is concluded that although Tac 2 and Tacr3 expression acts as puberty marker but increased NKB/NK3R signaling cannot lead to pubertal onset in mouse.

\section{Hypothalamic EAP1 (Enhanced at puberty involved in menstrual cyclicity in nonhuman primates}

Dissen et al. [1], designed an experiment to demonstrate the importance of EAP1 for normal reproductive cycle. EAP1 is considered as an important upstream regulator of neuroendocrine reproductive function in female hypothalamus. In this experiment, normal menstrual cycle of female monkeys was ceased due to inhibition of EAP1 expression in medial basal hypothalamus arcuate nucleus-region. It suggests the importance of this gene for pubertal onset. Although the cellular mechanism for EPA1 function is not fully understood but novel findings which describe its role as an integral component of a transcriptional repressor, suggest that it may regulate cyclicity by inhibiting downstream repressor genes.

\section{Variation in Lin28 gene is associated with age at menarche}

Tommiska et al. [4], found that variation in LIN28B is associated with timing of puberty according to genome-wide association (GWA) studies. LIN28B is a human ortholog of the gene that controls, through microRNAs, developmental timing in C. elegans. Moreover, Lin28a transgenic mice exhibit the puberty phenotypes. Thus, both LIN28B and LIN28A may have a role in pubertal development and are good candidate genes for monogenic ICPP. The study revealed that mutations in the coding region of LIN28B or LIN28A do not play a major role, if any, in the genetic etiology of ICPP. Further, it is confirmed that mutations in KISS1 and KISS1R are not common causes of ICPP in girls.

\section{Leptin gene (LEP) mutations result in abnormal puberty}

Murray et al. [10], reported that early onset of obesity, hypogonadism, pubertal delay and immune system abnormalities result due to various mutations in leptin gene. Constitutional delay in growth and puberty (CDGP) which is seen in paediatric clinics, in which children are present with delayed growth and puberty but usually also have a slim body habitus. The study was performed to test the hypothesis that LEP variants are responsible for appearance of CDGP phenotype. A novel missense variant (c.68COG) with proline to arginine substitution (p.P23R) was found in a child with CDGP. This sequence variant was not identified in any of the other control subjects but was identified in his mother who shared a similar phenotype of slim body habitus, reduced appetite and pubertal delay (menarche aged 15 years). The leptin variant showed similar stability in serum compared with wild type and did not demonstrate increased activity in an in vitro reporter 
gene assay. These were initial findings and it is hypothesized that this variant of leptin gene has increased bioactivity in vivo.

\section{Conclusion}

Puberty involves various events which are regulated by neuroendocrine hormonal pathways. Expression of multiple genes is involved in control of normal onset of puberty and successful reproduction. Some of the candidate genes include KISS1/Kiss1, Tac3, Tac3R, EAP1, Lin28 and LEP. Significance of these genes is evidenced by various mutations which lead to precocious or delayed puberty and other pubertal abnormalities. These neuronal and hormonal pathways are interconnected, and their regulation is controlled at various levels. Enormous amount of research has been done on puberty but still area of research is open for specific aspects including cellular and transcriptional level.

\section{References}

1. Dissen GA, Lomniczi A, Heger S, Neff TL, Ojeda SR (2012) Hypothalamic EAP1 (enhanced at puberty 1 ) is required for menstrual cyclicity in nonhuman primates. Endocrinology 153(1): 350-361.

2. Ojeda SR, Lomniczi A, Loche A, Matagne V, Kaidar G, et al. (2010) The transcriptional control of female puberty. Brain Res 1364: 164-174.

3. Silveira LG, Noel SD, Neto SAP, Abreu AP, Brito VN, et al. (2010). Mutations of the KISS1 gene in disorders of puberty. J Clin Endocrinol Metab 95(5): 2276-2280.
4. Tommiska J, Sørensen K, Aksglaede L, Koivu R, Puhakka L, et al. (2011). LIN28B, LIN28A, KISS1 and KISS1R in idiopathic central precocious puberty. BMC Research Notes 4: 363.

5. Witham EA, Meadows JD, Hoffmann HM, Shojaei S, Coss D, et al. (2013) Kisspeptin regulates gonadotropin genes via immediate early gene induction in pituitary gonadotropes. Mol Endocrinol 27(8): 1283-1294.

6. Mayer C, Boehm U (2011) Female reproductive maturation in the absence of kisspeptin/GPR54 signaling. Nat Neurosci 14(6): 704-710.

7. Popa SM, Moriyama RM, Caligioni CS, Yang JJ, Cho CM, et al.(2013) Redundancy in Kiss1 expression safeguards reproduction in the mouse. Endocrinology 154(8): 2784-2794.

8. Navarro VM, Pino RF, Garrido SMA, Galiano GD, Hobbs SJ, et al. (2012) Role of neurokinin $B$ in the control of female puberty and its modulation by metabolic status. J Neurosci 32(7): 2388-2397.

9. Gill JC, Navarro VM, Kwong C, Noel SD, Martin C, et al. (2012) Increased neurokinin $\mathrm{B}$ (Tac2) expression in the mouse arcuate nucleus is an early marker of pubertal with differential sensitivity to sex steroid-negative feedback than Kiss1. Endocrinology 153(10): 4883-4893.

10. Murray PG, Read A, Banerjee I, Whatmore AJ, Pritchard LE, et al. (2011) Reduced appetite and body mass index with delayed puberty in a mother and son: association with a rare novel sequence variant in the leptin gene. Eur J Endocrinol 164(4): 521-527.
Creative Commons Attribution 4.0 International License

For possible submissions Click Here

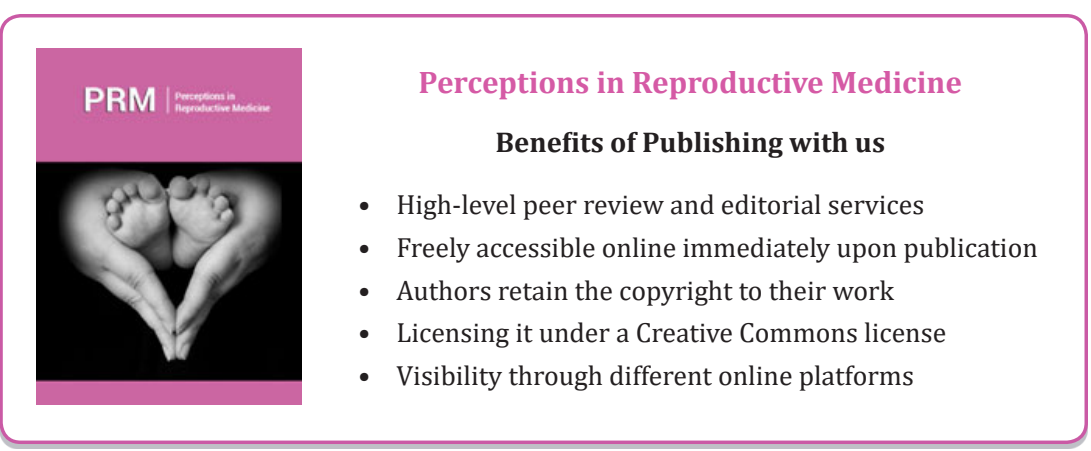

